\title{
Lipoxygenase Activation in Peanut Seed Cultivars Resistant and Susceptible to Aspergillus parasiticus Colonization
}

\author{
V. Müller, M. V. Amé, V. Carrari, J. Gieco, and R. Asis
}

First, second, and fifth authors: Departamento de Bioquímica/CIBICI, Facultad de Ciencias Químicas, Universidad Nacional de Córdoba, Haya de la Torre interseccion Medina Allende, Ciudad Universitaria, CP5000, Córdoba, Argentina; third author: Instituto de Biotecnología, Intituto Nacional de Tecnología Agropecuaria, Hurlingham, Buenos Aires, Argentina; and fourth author: Intituto Nacional de Tecnología Agropecuaria, Estación Experimental Manfredi, Córdoba, Argentina.

Accepted for publication 6 June 2014.

\begin{abstract}
Müller, V., Amé, M. V., Carrari, V., Gieco, J., and Asis, R. 2014. Lipoxygenase activation in peanut seed cultivars resistant and susceptible to Aspergillus parasiticus colonization. Phytopathology 104:1340-1348.

Accumulative evidence indicates that the lipoxygenase (LOX) pathway plays a significant role in the Aspergillus-seed interaction, such as interfering with activities of endogenous fungal oxylipins or producing antimicrobial compounds and signaling molecules. In this study, we characterized the LOX pathway in peanut seed during Aspergillus

resistant ('PI337394') and susceptible ('Florman INTA') to Aspergillus spp. infection and aflatoxin contamination. The LOX activity together with the content of LOX substrate and LOX products demonstrated the presence of a differential response mechanism to A. parasiticus infection between cultivars. Our findings suggest that this mechanism is under transcriptional control of previously identified (LOX 2 and LOX 3) and novel (LOX 4 and LOX 5) LOX genes. The results of this study support the role of these enzymes in defense during fungus infection in peanut seed.
\end{abstract} parasiticus colonization in a model of two cultivars distinguished as
The filamentous fungi Aspergillus flavus and A. parasiticus colonize oilseed crops (e.g., corn, peanut, cotton, and nut), causing important yield and economic losses. The infection produces not only tissue maceration but also aflatoxin contamination that constitutes an important health problem $(6,14)$.

Lipoxygenases (LOXs) (EC1.13.11.12) are nonheme iron-containing dioxygenases that are widely distributed among eukaryotes (8). These enzymes catalyze the addition of molecular oxygen at either the C-9 or C-13 residue of polyunsaturated fatty acids containing a cis, cis-1,4-pentadiene structure to produce an unsaturated fatty acid hydroperoxide $(22,35,42)$. Because most LOX isoforms preferentially catalyze one reaction over the other based on their regiospecificity, these enzymes are referred as 9-LOX or 13-LOX. Polyunsaturated fatty acids, particularly linolenic (C18:3) and linoleic (C18:2), can be oxidized by plant LOXs at either position 9 or 13 of their carbon chains (21). LOX primary products (fatty-acid hydroperoxides) are rapidly converted into a large class of oxygenated polyenoic fatty acids by at least six separate biosynthetic enzymatic pathways (17,35). All these metabolites, including fatty-acid hydroperoxides, are collectively called oxylipins. Diverse physiological functions have been proposed for oxylipins in plants, such as control of growth and development $(1,41)$, insect and pathogen resistance, (24) as well as tolerance to salt, drought, and cold stresses (25). Based on this evidence, the LOX pathway has been proposed to act directly in plant defense by producing antimicrobial compounds $(28,36)$ or

\section{Corresponding author: R. Asis; E-mail address: rasis@fcq.unc.edu.ar}

* The $\boldsymbol{e}$-Xtra logo stands for "electronic extra" and indicates that the online version contains three supplemental tables and three supplemental figures.

http://dx.doi.org/10.1094/PHYTO-12-13-0338-R

(c) 2014 The American Phytopathological Society by signaling molecules such as jasmonate that regulate gene expression of plant defense and cell death (30).

Accumulative evidence indicates that the LOX pathway plays a significant role in the Aspergillus-seed interaction (13). In this line, Zeringue et al. (43) have shown that volatile compounds derived from the LOX pathway inhibit Aspergillus spp. spore germination. Other authors demonstrated that $9 S$ - and $13 S$ hydroperoxyoctadecadienoic acid (HPODE) differentially affect Aspergillus spp. mycotoxin biosynthesis $(11,21)$, and these HPODE act as Aspergillus spp. sporulation factors (12). Further studies proposed that the primary products of plant 13- and 9-LOX (13S-HPODE and 9S-HPODE, respectively) modulate fungal developmental processes, including vegetative growth and sporulation $(9,38)$. The activity of these plant oxylipins was hypothesized to mimic or interfere with biological activities of endogenous fungal oxylipins.

Three LOX-encoding genes ( $L O X 1, \operatorname{LOX} 2$, and $L O X 3)$ have been reported in peanut thus far $(10,39)$. LOX 1 was shown to be highly expressed in flower and immature seed, whereas LOX 2 and LOX 3 were demonstrated to be highly expressed in mature seed. These three genes encode 13-LOX enzymes and respond differently against Aspergillus spp. infection. However, in these studies, the biochemical analysis of infected seed revealed a 9-LOX response to fungal infection, which is not fully explained by the expression of these genes.

Although considerable evidence suggests a role of the LOX pathway in response to Aspergillus spp. infection, it is not clear how this pathway is involved in either promoting the evolution of the infection or preventing it. Here, we investigated how the LOX pathway is regulated during $A$. parasiticus infection in peanut seed of two cultivars with contrasting behavior regarding susceptibility to A. parasiticus colonization and aflatoxin contamination, through biochemical and molecular analyses. The results herein presented support the role of LOX in defense during infection in peanut seed. 


\section{MATERIALS AND METHODS}

Plant material. 'PI337394' was discovered in northeastern Argentina and central Paraguay and introduced in the germplasm collection of the U.S. Department of Agriculture, and was characterized as resistant to Aspergillus spp. infection in field and in vitro seed infection assays $(4,29,32,34)$. 'Florman INTA' was obtained by a selection of 'Florunner' over 9 years and characterized as susceptible to Aspergillus spp. infection by in vitro seed infection assays (4). Peanut seed of two cultivars were provided by the Instituto Nacional de Tecnología Agropecuaria (INTA, Manfredi Experimental Station, Córdoba, Argentina).

The seed samples of the tested cultivars were obtained from the same growing season (2010-11). The cultivars were planted in a two-row plot, $10 \mathrm{~m}$ long, with an inter-row spacing of $0.70 \mathrm{~m}$ and 1 seed per $20 \mathrm{~cm}$ of linear row, in a completely randomized block design with two replicates. In turn, to eliminate all weeds, preplant (Imazetapir; $100 \mathrm{~cm}^{3}$ a.i./ha) and postemergence (Cletodin; $175 \mathrm{~cm}^{3}$ a.i./ha) herbicides were used. Leaf spot was avoided using contact fungicide (Mancozeb; $1 \mathrm{~kg}$ a.i./ha). Two complementary irrigations $(50 \mathrm{~mm})$ were applied in February and March to avoid drought stress. Each cultivar was manually harvested at its optimum maturity and threshed. Harvested pods, naturally dried to $5.5 \%$ moisture, were hand sorted to remove and discard visibly damaged pods, and stored in bags in a seed chamber at $7^{\circ} \mathrm{C}$ until use. Pods were hand shelled before experiments were performed.

Aspergillus spp. source. Isolate number 18 of A. parasiticus was used for all experiments. This isolate was previously determined as highly aflatoxigenic and infective in peanut seed (4). The fungi were grown on potato-dextrose agar (PDA) (Merck) at $30^{\circ} \mathrm{C}$ for 7 days. Spore suspensions were obtained by washing the surface of the cultures with Tween 80 (0.5 ml/liter) and quantified in a Neubauer chamber.

Infection assay and seed treatments. For all experiments, seed without tegument of both cultivars (PI337394 and Florman INTA) were surface sterilized with sodium hypochlorite. Disinfected seed (20 to 25 seeds/plate) were inoculated with $1 \mathrm{ml}$ of spore suspension of $A$. parasiticus $\left(1 \times 10^{4}\right.$ spores $\left./ \mathrm{ml}\right)$ and incubated at $30^{\circ} \mathrm{C}$ in petri dishes. Control seed received an equal amount of Tween 80 (mock-inoculated seed). Three independent biological replicates were conducted in parallel for all experiments.

Samples were collected at 5, 10, 20, 27, 48, and $72 \mathrm{~h}$ after inoculation (hai) for biochemical and molecular analyses.

Seed were examined by visual inspection to identify the presence or absence of mycelium on the seed surface; then, the percentage of colonized seed was calculated. Aflatoxin contamination was measured at 72 hai by high-performance liquid chromatography (HPLC) according to the procedure described by Asis et al. (4).

To conduct biochemical and molecular studies, seed were washed with $70 \%$ ethanol to eliminate the mycelium, frozen in liquid nitrogen, and kept at $-80^{\circ} \mathrm{C}$ until use. For biochemical analysis, seed were lyophilized for $16 \mathrm{~h}$ and then ground and defatted with chilled hexane $\left(-20^{\circ} \mathrm{C}\right)$ by using an Ultraturrax T18 basic (IKA Works Inc.). Defatted meals were used to measure the LOX activity and regiospecificity analysis. The oil obtained in the hexane extraction was used to measure the total hydroperoxides and free fatty acid contents.

Total hydroperoxides content and free fatty acids analyses in oils of infected peanut seed. Total hydroperoxides was analyzed according to Association of Official Analytical Chemists (AOAC) Official Method 965.33 (3), and defined as the milliequivalents of oxygen per $1,000 \mathrm{~g}$ of oil. Free fatty acids were determined by titration according to AOAC Official Method 940.28 (2) and expressed as grams of oleic acid in $100 \mathrm{~g}$ of oil.

LOX enzyme activity measurements. Seed LOXs were extracted from defatted meals with $0.2 \mathrm{M}$ phosphate buffer, $\mathrm{pH} 6.5$
(1:10, wt/vol), and precipitated with ammonium sulfate at $85 \%$ saturation for $1 \mathrm{~h}$, then centrifuged $\left(11,953 \times g, 15 \mathrm{~min}, 4^{\circ} \mathrm{C}\right)$. The precipitated proteins were dissolved in a minimum amount of $0.2 \mathrm{M}$ phosphate buffer, $\mathrm{pH} 6.5$, and desalted with DP 10 Sephadex G25 columns (GE Healthcare) to obtain the enzyme extract. An aliquot $(0.1 \mathrm{ml})$ of extract was mixed with $1.1 \mathrm{ml}$ of phosphate buffer $\left(\mathrm{pH} 6.5,0.2 \mathrm{M}\right.$ at $25^{\circ} \mathrm{C}$ ) and $0.8 \mathrm{ml}$ of substrate solution $(10 \mathrm{mM}$ linoleic acid) in a quartz cuvette. The absorbance $(234 \mathrm{~nm})$ was spectrophotometrically (Shimadzu UV 1601) monitored, using a time-scanning mode, and the reaction temperature was maintained at $25.0 \pm 0.5^{\circ} \mathrm{C}$. One unit of LOX was defined as the amount of enzyme which produces an increase in 0.001 absorbance unit per minute at $234 \mathrm{~nm}$ (37). The protein concentration of the enzyme extract was determined according to the Bradford method (7). The specific activity of LOX was expressed as LOX units per milligram of total protein.

Regiospecificity analysis: LOX product analyses by HPLCUV and gas chromatography mass spectrometry. To examine the composition of LOX products generated by enzyme extracts, $10 \mathrm{mM}$ linoleic acid (diluted in $0.2 \mathrm{M}$ sodium phosphate buffer, $\mathrm{pH}$ 6.5) was incubated with $0.5 \mathrm{ml}$ of enzyme extract for $30 \mathrm{~min}$ at room temperature. The reaction products were subsequently reduced to the corresponding hydroxy fatty acids by adding $2 \mathrm{ml}$ of $50 \mathrm{mM} \mathrm{SnCl}{ }_{2}$ and the reaction was stopped with $0.1 \mathrm{ml}$ of glacial acetic acid. The hydroxy fatty acids were extracted by adding $2 \mathrm{ml}$ of chloroform and the organic phase was recovered, dried, and reconstituted in $0.1 \mathrm{ml}$ of HPLC mobile phase (39).

HPLC runs were carried out on a Hewlett-Packard 1100 chromatograph, equipped with a UV detector and HP ChemStation Software. Absorbance at $234 \mathrm{~nm}$ (conjugated diene system of the hydroxy fatty acids) was recorded. Straight-phase HPLC of hydroxy fatty acid isomers was carried out on a Phenomenex Luna Silica column (150 by $4.6 \mathrm{~mm}, 5-\mu \mathrm{m}$ particle size) with a mobile phase of n-hexane:2-propanol:acetic acid $(100: 2: 0.1, \mathrm{vol} / \mathrm{vol} / \mathrm{vol})$ and a flow rate of $0.6 \mathrm{ml} / \mathrm{min}$. The elution times of 9-hydroxy-octadecadienoic acid (HODE) and 13-HODE were identified, and the fractions were collected and analyzed by gas chromatography mass spectrometry. Fractions were dried and derivatized with $\mathrm{N}, \mathrm{O}$-bis(trimethylsilyl)trifluoroacetamide and trimetilclorosilane (Supleco), then injected into a Varian $3800 \mathrm{Gas}$ Chromatograph coupled to a Varian Saturn 2200 Mass Spectrometer). The instrument was operated in the full-scan mode, in a range between 100 and $500 \mathrm{~m} / \mathrm{z}$. The 9-HODE and 13-HODE were characterized by a peak at $382 \mathrm{~m} / \mathrm{z}$ for the molecular ion $(\mathrm{M}+)$ and fragmentations represented by peaks at 311 and $225 \mathrm{~m} / \mathrm{z}$ (27).

Gene expression analyses. Total RNA was extracted from frozen seed with the plant RNeasy kit (Qiagen) according to the manufacturer's specifications, with minor modifications. Briefly, $80 \mathrm{mg}$ of tissue was macerated in liquid $\mathrm{N}_{2}$ using a mortar and pestle, transferred quickly to a $2-\mathrm{ml}$ tube, and vortexed vigorously with $600 \mu \mathrm{l}$ of RLT (kit buffer), including $10 \mu \mathrm{l}$ of $\beta$-mercaptoethanol per $1 \mathrm{ml}$ of buffer. The next steps were performed according to the manufacturer's recommendations, with the modification of incubating the samples for 5 min in Buffer RW1 (washing step) and recovering RNA three times, incubating for $5 \mathrm{~min}$ at $56^{\circ} \mathrm{C}$ in water. RNA was purified by $\mathrm{LiCl}$ precipitation $(2.25 \mathrm{M}$ final concentration) during $1 \mathrm{~h}$ at $-80^{\circ} \mathrm{C}$ and ethanol washed steps (39).

RNA samples were treated with DNaseI (Fermentas) to eliminate DNA contamination. A spectrophotometer (Synergy HT; Biotek Inc.) set at $260 \mathrm{~nm}$ was used for RNA quantification. Total RNA $(1 \mu \mathrm{g})$ was used as the template for reverse transcription and cDNA was synthesized using Revertaid M-MuLV Reverse Transcriptase (Fermentas), according to the manufacturer's instructions. RNA samples without reverse transcription were included as control (-RT).

Primers were designed by using the Primer Express (Applied Biosystems) software, based on sequences deposited in Gene 
Bank under the following accession numbers: gb|EZ722311.1 and gb|JR564445.1. In addition, a primer pair reported by Tsitsigiannis et al. (39) for indiscriminate expression analysis of peanut genes LOX 2 and LOX 3 was used. All primer used in this study are provided in Supplemental Table 1.

Quantitative polymerase chain reactions (PCRs) were carried out with a Bio-Rad iQ cycler with $1 \mu \mathrm{l}$ of cDNA (50 ng of reverse-transcribed total RNA), the SYBR Green PCR Master Mix spiked with fluorescein $10 \mathrm{nM}$ (Applied Biosystems), and $0.3 \mu \mathrm{M}$ primers in a final volume of $15 \mu$. The following amplification program was used: denaturation at $95^{\circ} \mathrm{C}$ for $3 \mathrm{~min}, 45$ cycles of amplification $\left(94^{\circ} \mathrm{C}\right.$ for $15 \mathrm{~s}, 61.3^{\circ} \mathrm{C}$ for $30 \mathrm{~s}$, and $72^{\circ} \mathrm{C}$ for $30 \mathrm{~s}$ ), and a melting curve program (from 52 to $62^{\circ} \mathrm{C}$, with an increment of $0.5^{\circ} \mathrm{C}$ for $5 \mathrm{~s}$ ). Reactions were performed in duplicate using cDNA templates from independent reactions for each LOX and reference gene. Amplicon specificity was confirmed based on the dissociation curve produced at the end of each run and on visualization of the products, following electrophoresis on $2.5 \%$ agarose gel.

To determine gene-specific PCR amplification efficiency, a five-dilution series of the samples at different time references of infection was constructed. Based on the cycle threshold values for all dilution points in a series, a standard curve was generated using linear regression. The gene-specific PCR amplification efficiency was calculated using the following equation: efficiency $\%=\left(10^{(-1 / \text { slope })}-1\right) \times 100(31)$.

The histone $\mathrm{H} 3$ encoding gene $(H 3)$ was used as a reference gene, according to expression stability for normalization of gene expression (31). To determine the relative expression ratio of the $L O X$ genes at each treatment, the normalized $L O X$ RNA value was divided by the calibrator value.

To confirm the specific gene expression, the PCR products were sequenced with GeneAmp PCR System 9700 (Applied Biosystems) and 3130xl Genetic Analyzer (Applied Biosystems) using Hidroshear (Genomic Solutions).

Statistical analyses. Statistical analyses were carried out using the Infostat Software Package (26). All values are expressed as means \pm standard deviation. Normal distribution of data was analyzed by the Shapiro-Wilk test, and the Levene test was used to check homogeneity of variance. All data were subjected to analysis of variance (ANOVA). When significant differences were found by ANOVA, the Fisher test was used for mean separation. When data were not normally distributed, data were subjected to nonparametric statistical analysis on ranks (Kruskal-Wallis), followed by Dunn's posttest (15). Significance was accepted at $P<$ 0.05 for all comparisons. For integrative data analysis, principal component analysis (PCA), Pearson's correlation, and cluster analysis were performed. Data were processed as [ $\log _{10}$ (infected/ mean of control)]. For clustering analysis, the average linkage method was used and $50 \%$ of Euclidian distance was defined to set the clusters.

\section{RESULTS}

Seed infection. We have previously reported different susceptibility to $A$. parasiticus and A. flavus infection and aflatoxin contamination among six public cultivars (4). Particularly, we characterized a resistant (PI337394) and a susceptible (Florman INTA) peanut cultivar to both infections and aflatoxin production. To confirm that observation, we conducted an A. parasiticus infection assay and found that these cultivars constitute a suitable model system to understand the biochemical and molecular mechanisms underlying the peanut seed-necrotrophic pathogen interaction. Fungal infection was observed at 5, 10, 20, 27, 48, and 72 hai and aflatoxin was measured at 72 hai. From 48 hai, mycelium was observed on $50 \%$ of Florman INTA seed and, after 72 hai, all seed were infected. By contrast, no infection or mycelial growth was observed on PI337394 until 72 hai, when only
$10 \%$ of the seed were colonized (Supplemental Figure 1). Correspondingly, aflatoxin content was higher in Florman INTA than in the resistant cultivar $(169 \pm 50$ versus $3.7 \pm 2 \mathrm{ppb}$ of total aflatoxin for Florman INTA and PI337394, respectively; $P<0.001$ ).

LOX activity and regiospecificity. To evaluate the relation of A. parasiticus infection response and LOX enzyme activity, we measured LOX activity in our experimental system in a time series of infected and control peanut seed (Supplemental Table 2). Although the two cultivars showed different patterns of LOX activity during the incubation period, significant differences between infected and control seed were found at 20 hai in both cultivars. At that time, PI337394 showed a significant increase of LOX activity in infected seed, in comparison with the mockinoculated control. Contrastingly, infected seed from Florman INTA showed significant decreases in LOX activity in relation to mock-inoculated seed. Average changes of LOX activity between infected and control seed ranged between +3.3 - and -6.5-fold (at 20 hai) for the resistant (PI337394) and susceptible (Florman INTA) cultivars, respectively (Fig. 1A).

The regiospecificity of LOX enzyme extract of peanut seed at each infection time was determined by measuring the hydroperoxide isomers (9- and 13-HPODE) formed in the enzymatic oxidation of linoleic acid. The composition of 9- and 13-HOPDE is shown in Figure 1B for PI337394 and Florman INTA. Significant differences were found at 10 hai between isomers from infected and control seed of PI337394, where a significant increase of 9-HPODE percentage was observed. No significant differences in HPODE isomer composition were found between infected and control seed of Florman INTA. However, significant differences were detected between control seed of both cultivars at 10 hai (Florman INTA: 41 and 59\%, and PI337394: 58 and $42 \%$, for 9- and 13-HPODE, respectively) and at 20 hai (Florman INTA: 56 and 44\%, and PI337394: 42 and 58\%, for 9- and 13-HPODE, respectively). Similar differences were detected between infected seed of both cultivars at 10 hai (Florman INTA: 45 and 55\%, and PI337394: 83 and 17\%, for 9- and 13-HPODE, respectively) and at 48 hai (Florman INTA: 50 and 50\%, and PI337394: 64 and 36\%, for 9- and 13-HPODE, respectively).

Based on these and previous, results, it is apparent that the two cultivars used in this study responded differently to A. parasiticus infection by regulating the LOX pathway, not only in relation to enzyme activity but also regarding the LOX regiospecificity.

Free fatty acid and total hydroperoxides analysis in infected peanut seed. In order to estimate LOX substrate availability in peanut seed after $A$. parasiticus infection, free fatty acid contents of seed oil were measured at different infection time references. Significant differences of free fatty acid contents were found between infected and control seed in PI337394 at 10 and 27 hai whereas, for the susceptible cultivar, these differences were found at 10, 20, and 72 hai. Fold changes of acidity were also calculated to show the extent of free fatty acids modification by the A. parasiticus infection (Fig. 2A). Florman INTA showed a significant upregulation at 10 and 72 hai and a significant downregulation at 20 and 48 hai. By contrast, PI337394 showed a significant upregulation at 27 hai and a significant downregulation at 10 hai.

Fatty acid hydroperoxides are the main products of LOX enzyme activity. To estimate the contents of these products during A. parasiticus infection in both cultivars, we measured hydroperoxide values in seed at different infection times. PI337394 showed significant differences of hydroperoxide content between infected and control seed at 10 and 20 hai, whereas Florman INTA only showed a significant difference at 10 hai. Fold changes of hydroperoxide values between infected and control seed are shown in Figure 2B. Upregulation of hydroperoxide values was found at 10 and 20 hai in Florman INTA and at 27 hai in PI337394, whereas a downregulation of hydroperoxide levels was found at 27 hai in Florman INTA and at 10 and 20 hai in PI337394. 
The study of free fatty acids and total hydroperoxides showed a differential response of peanut cultivars to A. parasiticus infection that was detected between 10 and 27 hai, a time period in which LOX activities showed the major differences between cultivars.

Gene expression. To identify genes encoding LOX enzymes responsible for the biochemical shift observed during $A$. parasiticus infection, $L O X$ unigene sequences were searched in the Transcriptome Shotgun Assembly Sequence Database (http:// www.ncbi.nlm.nih.gov/genbank/tsa). Two sequence records, $\mathrm{gb} \mid$ EZ722311.1 and gb|JR564445.1 (named in this report as LOX 4 and $L O X$ 5, respectively), were selected and analyzed. The predicted $L O X 4$ and $L O X 5$ open reading frames encode proteins of 863 and 860 amino acids, respectively. Both harbor short amino terminal PLAT-LH2 domains and a major C-terminal catalytic LOX superfamily domain (Supplemental Figure 2). Neither LOX4 nor LOX5 were predicted to contain signal or transit peptides by PSORT and TargetP. These observations suggest that they are likely localized in the cytosol (data not shown).

In addition to these genes, we selected two previously reported peanut $L O X$ genes ( $L O X 2$ and $L O X 3$ ) (39) harboring the same domains described for $L O X 4$ and $L O X 5$. All selected $L O X$ genes were analyzed phylogenetically, according to the previously reported analysis used for LOX 2 and LOX 3 (39) (Supplemental Figure 3). The phylogenetic tree showed peanut LOX 4 in the cluster of leguminous LOX and fell into the type I 13-LOX clade together with the peanut LOX 1, 2, and 3 paralogs. However, LOX 4 was more closely related to Glycine max LOX 4 (BAA03101), 5 (AAB67732), and 8 (AAC49159) than to peanut LOX 1,2 , and 3 . In fact, LOX 4 showed $80 \%$ identity (coverage

A

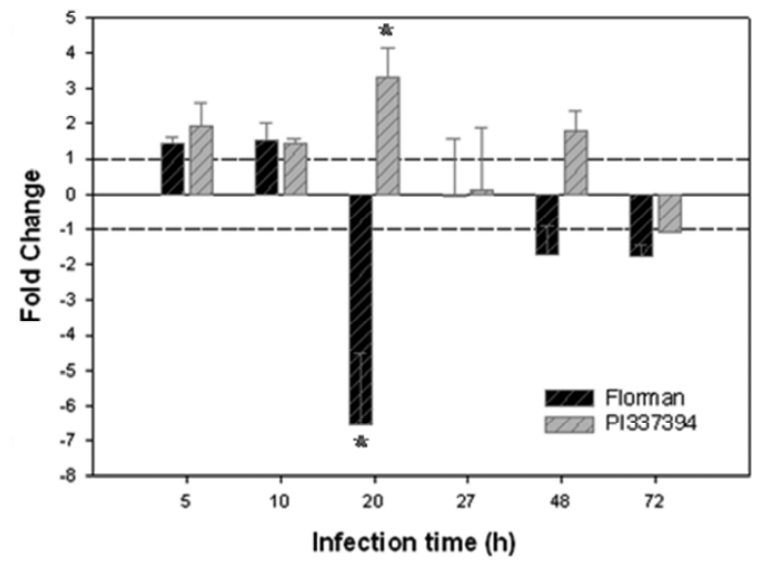

98\%) with G. $\max$ LOX 4 and 8 and $78 \%$ identity with G. max LOX 5. On the other hand, peanut LOX 5 fell into the type I 9LOX cluster closely related to Prunus dulcis LOX 1 (CAB94852) (72\% identity, coverage $100 \%$ ) and, to a lesser extent, Arabidopsis LOX 1 (AAA32827) (68\% identity, coverage 100\%).

To examine the expression pattern of LOX in response to $A$. parasiticus infection, $\operatorname{LOX} 2,3,4$, and 5 genes were analyzed in infected and control peanut seed of both cultivars at postinfection time references. For LOX 2 and 3 genes, expression was analyzed with the primer set reported by Tsitsigiannis et al. (39) that anneals indiscriminately on both genes in Arachis hypogaea and thus, because of this characteristic, the expression of these genes is mentioned as LOX 2-3. Because all biochemical changes occurred before 48 hai, gene expression analyses were conducted until this time reference. Different expression patterns between cultivars can be seen in Supplemental Table 3 and Figure 3. LOX 2-3 expression in susceptible Florman INTA showed significant differences between infected and control seed at 5, 10, and 48 hai, when infected seed showed higher expression. The fold-change analysis indicates that LOX 2-3 in Florman INTA is upregulated in infected seed at 5 and 10 hai followed by a downregulation at 20 hai and a smooth upregulation up to 48 hai (Fig. 3A). By contrast, in the resistant cultivar, the expression of this gene showed significant differences between infected and control seed at 5, 20, 27, and 48 hai. Fold-change analysis of LOX 2-3 in PI337394 showed an upregulation during infection at 5 and 10 hai, followed by a strong downregulation up to 48 hai (Fig. 3A)

$L O X \quad$ expression analyses showed a pattern of expression similar to $L O X$ 2-3 genes in infected and control seed of Florman INTA. However, the LOX 4 pattern in PI337394 was different

\section{B P1337394 cultivar}
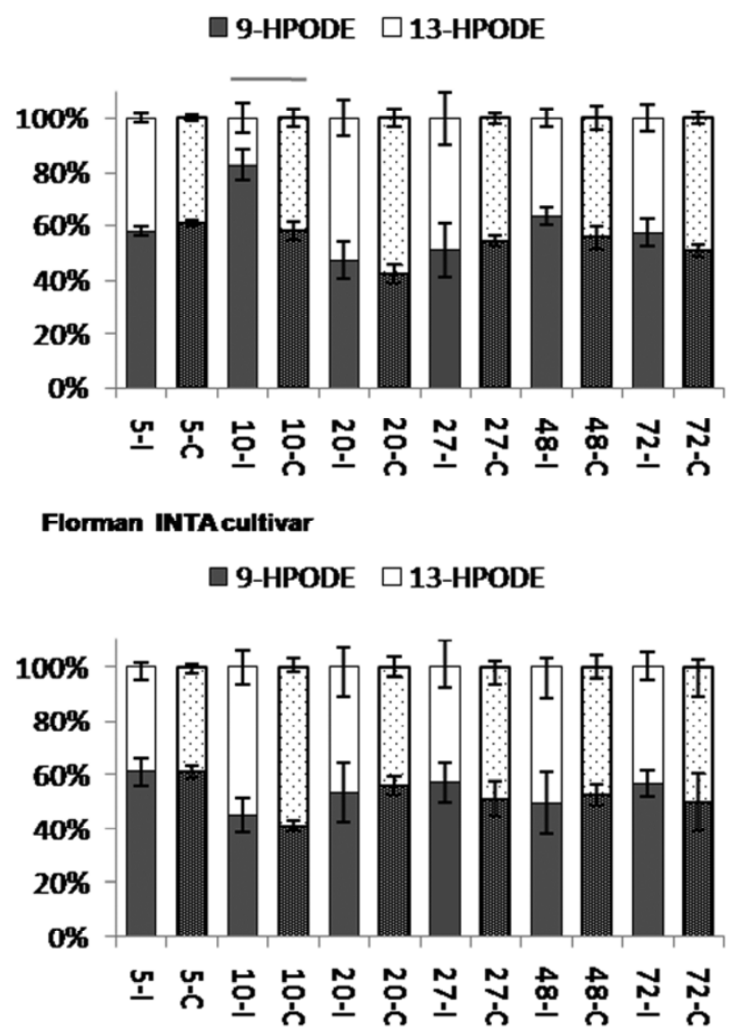

Fig. 1. Lipoxygenase analysis in peanut seed infected by Aspergillus parasiticus. A, Lipoxygenase activity fold change of infected seed with respect to the average value of control (mock-inoculated seed) of PI337394 and Florman INTA. Values between 1 and -1 indicate no change with respect to the control; an asterisk (*) indicates significant change $(P \leq 0.05)$ between infected and control seed. B, Hydroperoxyoctadecadienoic acid (HPODE) isomer composition produced by the reaction of peanut seed lipoxygenases from different infection times and linoleic acid as substrate. HPODE isomer composition is expressed as percentage of 9-HPODE and 13-HPODE. C = control seed (dot bars) and I = infected seed. Horizontal line above the bars indicates significant difference between infected and control seed. 
from that of $L O X$ 2-3; whereas $L O X 4$ was upregulated during the infection from 10 to 20 hai, its expression then started to decline gradually up to 48 hai (Fig. 3B).

The pattern of expression of the LOX 5 gene was the most different, in comparison with the other LOX genes. In Florman INTA, LOX 5 showed no difference between infected and control seed up to 20 hai, whereas a significant increase in infected seed was found at 27 hai. By contrast, in Pi337394, LOX 5 expression showed significant differences between infected and control seed from 5 to 20 hai, which indicates an upregulation during the infection in this time period (Fig. 3C).

Integration of metabolic and gene expression responses. To better understand the coordination of LOX gene expression in the context of the biochemical responses, we performed PCA to identify differences in the response to fungal infection between cultivars (Fig. 4). Cultivar response was distinctly discriminated by the first $(52.3 \%$ of total variance explained) and second (47.7\% of total variance explained) principal components, where the Florman INTA response was mainly associated with $L O X$ 2-3 expression, total hydroperoxide content, and LOX 2-3/LOX 5 expression ratio and, to a lesser extent, to LOX 2-3/LOX 4 expression ratio. Conversely, the PI337394 response was mainly associated with $L O X$ 5/LOX 4 expression ratio, LOX activity, $\% 9 H P O D E$, and LOX 5 expression.

Pearson's correlation analysis was applied in order to find possible links between gene expression and the activities of their products in each cultivar and across the time course of infection. All variables were clustered using average linkage clustering and depicted along a heat map showing the significant Pearson's correlations (most correlations were significant at $P \leq 0.05$ but an asterisk [*] was used in cases where significance was determined at $P \leq 0.1$ in Figure 5). Two clusters were observed in the response of INTA Florman (Fig. 5A): cluster I and II. Cluster I links LOX 2-3, LOX 2-3/LOX 4 expression ratio, LOX 2-3/LOX 5 expression ratio, and $\operatorname{LOX} 4$, which is in line with PCA. Interestingly, these variables of cluster I correlated positively with most of the biochemical variables, such as free fatty acids ( $L O X$ $2-3, P=0.01 ; L O X 2-3 / L O X 5$ ratio, $P=0.02$ ) and total hydroperoxide content ( $L O X 4, P=0.09$ ). By contrast, $L O X$ 5/LOX 4 expression ratio (cluster II) correlated negatively with LOX activity $(P=0.01)$ and free fatty acids $(P=0.004)$, In addition,
$L O X 4$ expression correlated positively with $L O X \quad 2-3$ expression $(P=0.02)$ and LOX 5 expression $(P=0.05)$, as well as free fatty acid $(P=0.01)$ and total hydroperoxide contents $(P=0.09)$.

In the response of PI337394, four clusters of variables were found (Fig. 5B). Cluster I links most of the variables associated with PI337394 response in PCA and correlated with all the biochemical variables. In this cluster, the LOX 5/LOX 4 expression ratio correlates positively with free fatty acids $(P=0.02)$, total hydroperoxide content $(P=0.01)$, and percentage of 13HPODE $(P=0.04)$, whereas $L O X 5$ expression correlates positively with LOX activity $(P=0.1)$.

Taking all these results into account, we believe that the observed biochemical changes in the LOX pathway in response to Aspergillus infection are under transcriptional control of the LOX encoding genes in peanut seed.

\section{DISCUSSION}

Plant LOX enzymes are thought to play an important role in several physiological processes such as pollination, signaling, and pathogen-defense responses $(17,30)$.

In a previous study, we evaluated the susceptibility to aflatoxin contamination in seed of three cultivars with known Aspergillus spp. susceptibility (PI337394, 'PI337409', and 'J11') (29,32,34), along with a group of cultivars developed locally (National Institute of Agricultural Technology of Argentina: 'Florman', 'Manfredi 68', and 'Colorado Irradiated') (4). In that study, seed infection assays were conducted using 21 isolates of Aspergillus flavus and A. parasiticus obtained from Argentine peanut production areas. It was concluded that resistance to aflatoxin contamination is linked to resistance to fungal infection, specifically in the seed integument and cotyledons. In particular, Florman INTA showed the highest susceptibility to infection and a high susceptibility to aflatoxin contamination and PI337394 showed a reduced infection and aflatoxin contamination.

In the present study, seed without integuments from these two cultivars also showed this behavior. By using these two cultivars as a model, we investigated how the LOX pathway is regulated during A. parasiticus infection through biochemical and molecular analyses. We evaluated LOX enzymatic activity in infected and control seed and found an early response (during the first
A

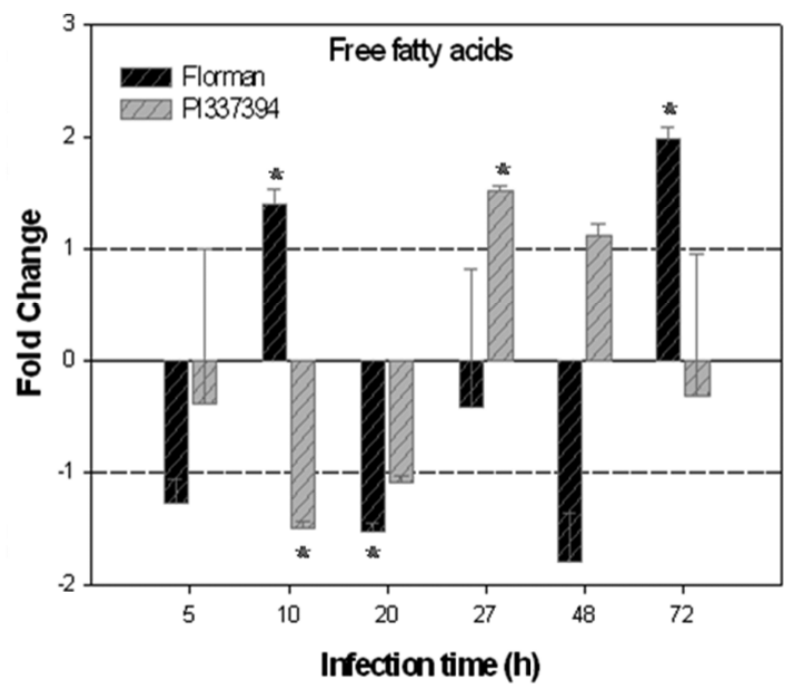

B

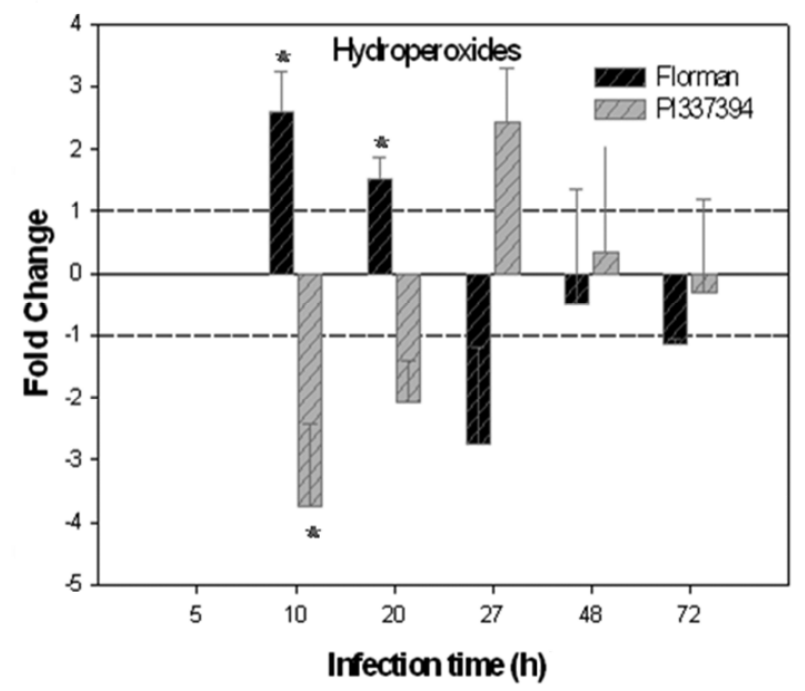

Fig. 2. Free fatty acids and hydroperoxide content in peanut seed infected by Aspergillus parasiticus. A, Fold change of free fatty acid content of infected seed with respect to the average value of control seed of PI337394 and Florman INTA. Values between 1 and -1 indicate no change with respect to the control. B, Fold change of hydroperoxide content of infected seed with respect to the average value of control seed of PI337394 and Florman INTA. Values between 1 and -1 indicate no change with respect to the control; an asterisk $(*)$ indicates significant change $(P \leq 0.05)$ between infected and control seed. 
20 hai) of this activity to fungal infection (Fig. 1). Noticeably, at 20 hai, the resistant cultivar presented the highest activity and response to infection (3.3-fold change), whereas the susceptible cultivar showed the opposite response at the same time after inoculation (-6.5-fold change). In a similar study, Burow et al. (10) found a strong downregulation of LOX activity after $48 \mathrm{~h}$ of A. parasiticus infection in seed from a susceptible peanut cultivar (Florunner). Moreover, the pattern of LOX activity reported by these authors resembles the activity showed by the susceptible cultivar of our model system (Florman INTA).

In parallel, we evaluated the free fatty acid content as an estimation of lipase activity in seed (Fig. 2A). It has been reported that phospholipases are activated by biotic and abiotic stress and could act as triggers of the LOX pathway, releasing unsaturated fatty acids (mainly linoleic and linolenic) from plasma membranes that are susceptible to the action of LOX in the cytoplasm and plastids (40). The findings presented in the current study showed that free fatty acid content varies significantly between 10 and 27 hai in response to infection and also showed the presence of an inverted pattern between cultivars (Fig. 2A). After this period of time, changes in free fatty acid content of seed could be attributable to the presence of fungal lipases. Although no lipase activity was directly measured, we can hypothesize that, in these cultivars, lipases respond differently to infection stimulus. The content of free fatty acids in Florman INTA significantly correlated with LOX activity and $L O X$ gene expression ( $L O X$ 2-3/LOX 5 ratio and LOX 2-3) during infection (Fig. 5A). However, in PI337394, significant correlations of free fatty acid content with LOX genes (LOX 5/LOX 4 and LOX 4) and hydroperoxide content was observed (Fig. 5B). The present results suggest a signaling mechanism between lipase activity and LOX activity and LOX gene expression in response to infection, which operates differentially between cultivars.

In addition to studying the availability of LOX substrate, we analyzed the LOX products formed in the seed of both cultivars during $A$. parasiticus infection, using different approaches. First, total hydroperoxide content was measured (Fig. 2B). As in the acidity analysis, significant changes in hydroperoxide production were found between 10 and 27 hai in both cultivars in response to the infection. However, the pattern of the hydroperoxide content was different from that of free fatty acid production when the cultivars were compared (Fig. 2A and B). By contrast, as expected, the LOX activity and total hydroperoxide content showed a similar response pattern (Figs. 1A and 2B).

Moreover, slight differences in the response time could be observed with LOX, preceding hydroperoxide content changes. Because 9- or 13-hydroperoxide yield by plant LOXs depends on enzyme regiospecificity, then the composition of HPODE produced in seed of both cultivars was estimated with the reaction of linoleic acid with enzyme extracts from different infection times (Fig. 1B). The major and significant change in relation to control was observed at 10 hai in resistant PI337394, where HPODE composition showed an increase in the proportion of 9-HPODE $(83 \%)$ in response to infection (Fig. 1B, upper). At this time, an increase in the proportion of 13-HPODE was also detected (59\%) in control and infected seed of susceptible Florman INTA (Fig. 1B, lower). These results showed a predominant activity type 9-LOX in the resistant cultivar and an activity type 13-LOX in the susceptible cultivar. At 20 hai, an increase in the proportion of 13HPODE (53\%) was observed in PI337394. This increase was gradually reduced after 48 hai $(36 \%)$. Meanwhile, the proportion of 13-HPODE in Florman INTA was reduced to $46 \%$ after 20 hai and remained at that rate up to 72 hai. Both polyunsaturated fatty acids (linoleic and linolenic) as well as 9- and 13-HPODE are signaling molecules which have significant effects on plant defense and in fungi differentiation process (13). Calvo et al. (12) reported that $\mathrm{C} 18$ fatty acids and 9-HPODE promoted sporulation in A. flavus, A. parasiticus, and A. nidulans and also stimulated aflatoxin formation. Conversely, 13-HPODE inhibited the toxin synthesis (11). Another study showed that maize 9-LOX ( $Z m L O X$ $3)$, engineered into the conidia-deficient $A$. nidulans mutant DppoAC, was able to restore conidia production (9). Based on genetic evidence, these authors proposed a reciprocal oxylipin crosstalk in the Aspergillus-seed pathosystem. However, a knockout mutant of the maize type 9-LOX gene (ZmLOX 3) was shown to be more susceptible to $A$. flavus and had significantly higher levels of aflatoxin contamination than nonmutant lines both in kernel assays and under field conditions (20). The results from this last report are in line with our results, where a 9-LOX response to $A$. parasiticus infection is associated with the resistant
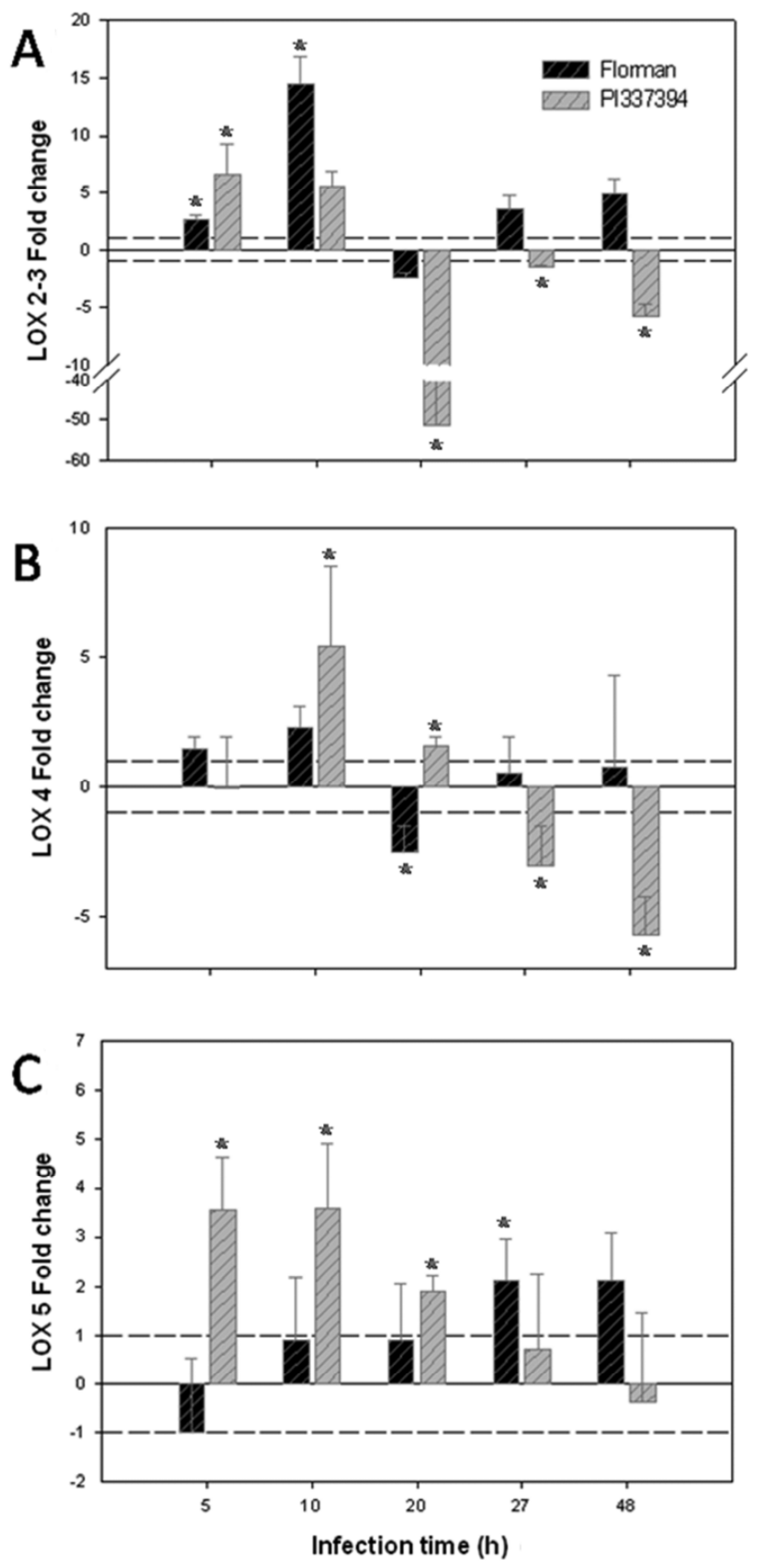

Fig. 3. Quantitative reverse-transcription polymerase chain reaction analysis of $L O X 2-3, L O X$ 4, and LOX 5 genes in PI337394 (correspond to gray bars) and Florman INTA (correspond to black bars). LOX expression are represented as fold change of infected seed with respect to the average value of control seed corresponding to the same time after inoculation. Values between 1 and -1 indicate no change with respect to the control. A, Fold change of LOX 2-3. B, Fold change of $L O X 4$. C, Fold change of LOX 5. An asterisk (*) indicates significant change $(P \leq 0.05)$ between infected and control seed. 
cultivar. All these results support the conclusion that plant oxylipins are implicated, to some extent, in plant-fungus interactions. Nevertheless, the observed effects in Aspergillus spp. by exogenous application of pure oxylipins to culture medium are not so straightforward in the seed.

In an attempt to elucidate the transcriptional control of LOX activity, we analyzed the expression of the genes $L O X 2-3,4$, and 5. LOX 2-3 corresponds to two almost identical $L O X$ gene alleles (LOX 2 and LOX 3) highly expressed in mature seed (39). The other two (LOX4 and LOX 5) correspond to two peanut unigenes deposited in GenBank as gb|EZ722311.1 and gb|JR564445.1, respectively. LOX 2 and 3 genes encode type 13 LOXs with a regiospecificity against linoleic acid of $84 \%$ of $13-\mathrm{HOPDE}$ and $16 \%$ of 9-HOPDE (39). LOX 4 encodes a type 13 LOX which is closer to $G$. max LOX 4 (BAA03101), G. $\max$ LOX 5 (AAB67732) and G. max LOX 8 (AAC49159) than to peanut LOX 2 and 3. These soybean LOXs have shown to have high affinity to free linoleic and linolenic acids, but not to their esterified forms, and to produce a ratio of 60:40 of 13- and 9-HPODE-derived from linoleic acids (19). G. max LOXs are associated with vegetative and reproductive tissue (18) but also have been shown to be involved in nodule development and pod defense $(16,23)$. LOX 5 encodes a type 9 LOX that is closely related to $P$. dulcis LOX1 (CAB94852) and Arabidopsis thaliana
LOX1 (AAA32827), two proteins described to form exclusively 9-HPODE $(5,33)$ and widely associated with plant defense (41).

The expression analysis of peanut $L O X$ genes (Fig. 3) showed a different expression pattern in response to Aspergillus parasiticus infection when both cultivars were compared. Both type 13 LOX genes (LOX 2-3 and LOX 4) showed a similar pattern response to A. parasiticus infection in each cultivar. Interestingly, whereas both genes responded early to infection, followed by a downregulation at 20 hai in both cultivars, after this period of time, a different response between cultivars was observed (Fig. 3A and B). A previous study also reported a strong downregulation of LOX 2-3 expression after A. flavus infection in peanut seed, during the infection period (39). These authors also reported that A. nidulans dioxigenase mutants decreased the expression of peanut LOX 2-3 genes during infection compared with the wild type, suggesting a cross-talk between fungus and seed through dioxigenase activities (9). Moreover, in another work, it was reported that this mutant showed a reduced ability to colonize peanut seed (38). All these results suggest that repression of peanut $L O X 2$ and $L O X 3$ genes is required to lower seed fungal colonization. Our results support this hypothesis because the resistant cultivar showed a drastic repression of LOX 2-3 after 10 hai, whereas the susceptible one showed a mild repression followed by an upregulation (Fig. 3A).

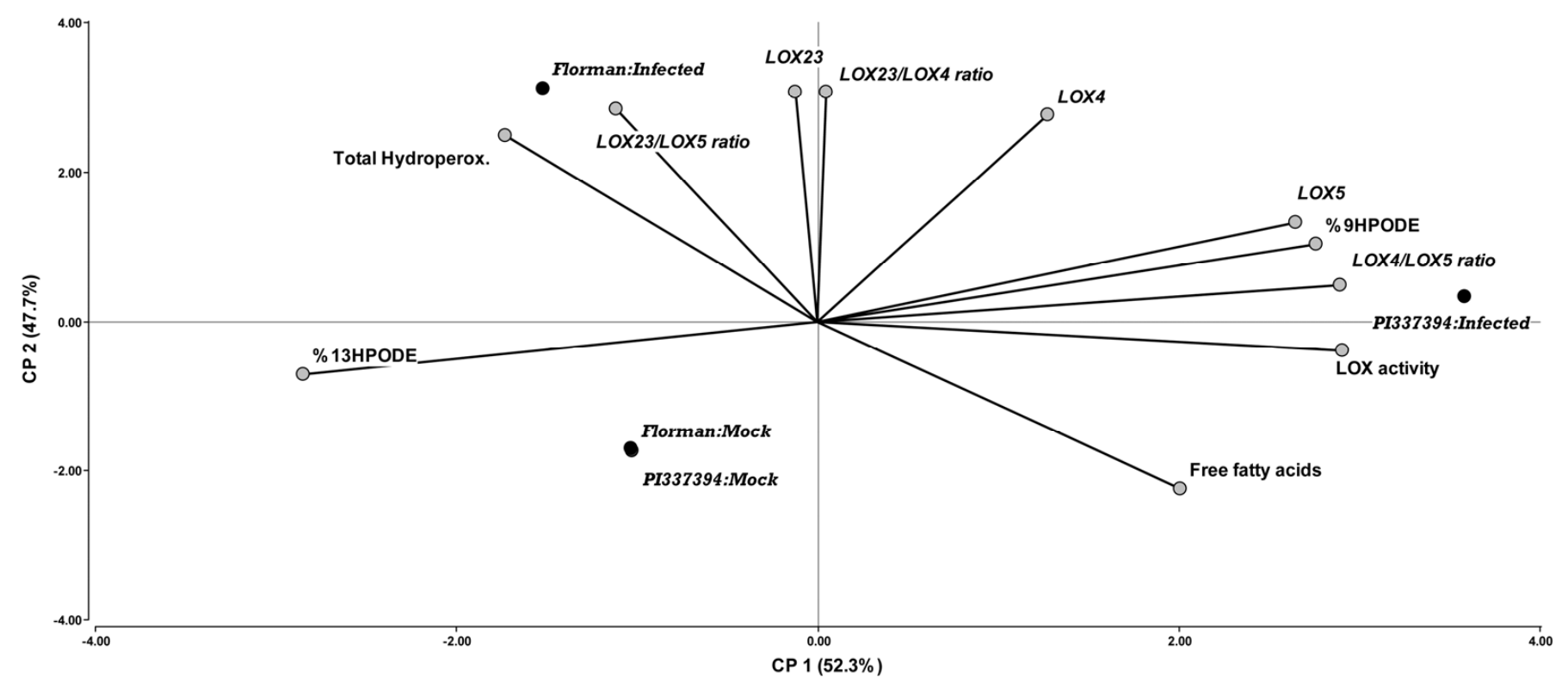

Fig. 4. Biplot for principal component analysis of biochemical and gene expression response to Aspergillus parasiticus infection of two peanut cultivars.
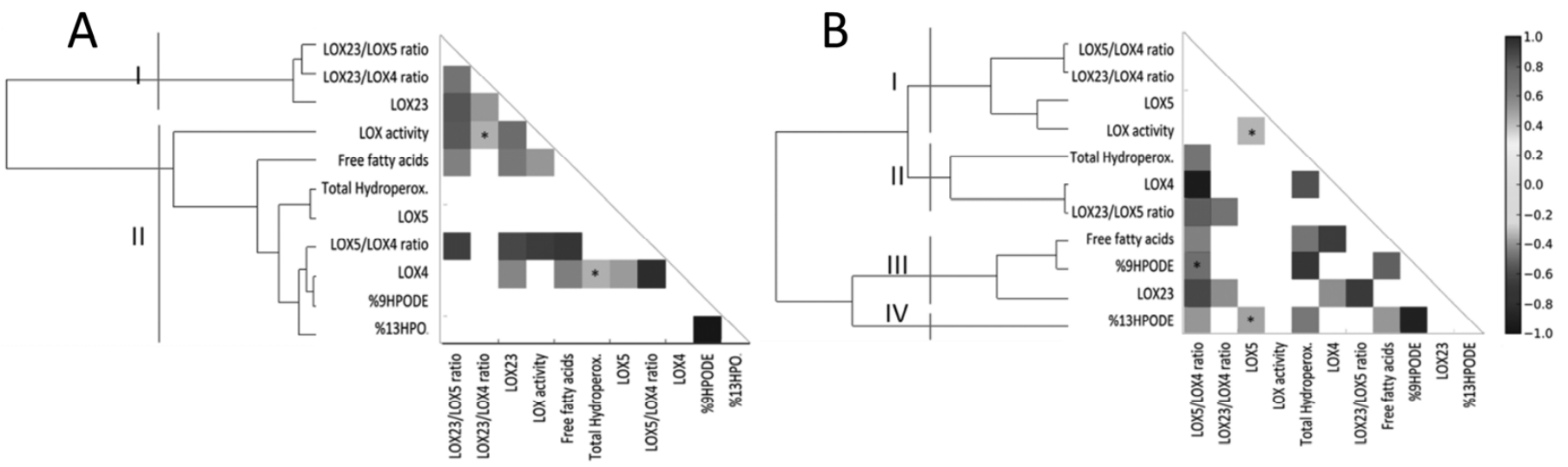

Fig. 5. Average linkage clustering graphs and heat maps of significant Pearson's correlations $(P \leq 0.05$, * indicates $P \leq 0.1)$ of biochemical and genetic shifts during Aspergillus parasiticus infection in A, Florman INTA seed and B, PI337394 seed. Color scale bar on the right shows correlation coefficient values. 
Alternatively, evidence of previous studies support the hypothesis of a positive association between type 9-LOX activity and seed fungal colonization, based on the induction of a peanut LOX 1 gene (a putative type 9-LOX) after Aspergillus spp. infection (10) and the effects of 9-LOX products on differentiation processes of Aspergillus spp. that contribute to promote seed colonization $(11,12)$. However, our results showed an early response of the LOX 5 gene (a putative type 9-LOX) to A. parasiticus infection in the resistant PI337394 and a later response in the susceptible Florman INTA (Fig. 3C), which is contradictory to the previous hypothesis.

In order to elucidate the response of both cultivars to A. parasiticus infection, their genetic and biochemical changes were integrated. This analysis showed that both cultivars present a different coordinated transcriptional control in response to $A$. parasiticus infection. In the resistant cultivar, the LOX 5/LOX 4 expression ratio seems to drive the biochemical change of LOX pathway variables (Figs. 4 and 5B). In this cultivar, LOX 5 expression is upregulated early in the infection process, probably producing the observed increase of 9-HPODE at 10 hai (Fig. 1B). In addition, $L O X 4$ is subsequently upregulated with a maximum expression at 20 hai, giving together the maximum LOX activity response at this time $(20 \mathrm{~h})$ and a maximum of total hydroperoxide content at 27 hai. By contrast, the biochemical change of LOX pathway in the susceptible cultivar seems to be driven for the $L O X \quad 2-3 / L O X \quad 5$ expression ratio and $L O X \quad 2-3 / L O X \quad 4$ expression ratio (Figs. 4 and 5A). LOX 2-3 and LOX 4 expression is upregulated early for infection, followed by a significant repression at 20 hai, strongly correlating with LOX activity and total hydroperoxide content (Fig. 5A), and could be associated with the increase in 13-HPODE synthesis displayed in the enzyme extract of seed sampled at 10 hai (Fig. 1B).

The results presented in this study indicate a different action of the LOX pathway in seed of two peanut cultivars challenged by A. parasiticus infection. An adaptation to resist fungal infection seems to be associated with a production of 9-HPODE mediated by the expression of type $9-L O X$ gene, as was previously reported for maize $L O X$ 3. LOX 3 is also a 9 LOX that was shown to be required for defense against $A$. flavus and $A$. nidulans by knockout mutant analyses (20). Considering this, we report here two novel peanut $L O X$ ( $L O X 5$ and $L O X 4$ ) genes whose levels of expression may explain the changes in LOX activities. In this adaptation to resist fungal infection, we observed an early 9HPODE production driven for a regulated coexpression of $L O X 5$, predominantly on $L O X 2-3$ and $L O X \quad 4$. By contrast, an early response of exclusively type $13-\mathrm{LOX}$ mediated by the co-expression of $L O X 2-3$, predominantly on $L O X 5$ and $L O X 4$, seems to promote the infection. Other LOXs could be participating in the response to infection in addition to the two novel $L O X$ gene products, which would fully explain all reported metabolic changes.

Based on the results of this study, we believe that A. parasiticus resistance in peanut seed is closely related to a rapid onset of defense mechanisms. In a preliminary study, we identified antifungal oxilypins produced by the resistant cultivar in response to A. parasiticus infection (unpublished results). The characterization of this antifungal production in both cultivars, together with other metabolic branches of oxylipin biosynthesis, are needed to fully understand how LOX pathways are related to peanut seed resistance to $A$. parasiticus infection and aflatoxin contamination.

\section{ACKNOWLEDGMENTS}

V. Müller was recipient of a fellowship of Consejo Nacional de Investigaciones Científicas y Técnicas (Argentina). M. V. Amé, F. Carrari, and R. Asis are members of Consejo Nacional de Investigaciones Científicas y Técnicas (Argentina). This work was partially supported by the Instituto Nacional de Tecnología Agropecuaria, Consejo Nacional de Investigaciones Científicas y Técnicas, and Agencia Nacional de Promoción Científica y Tecnológica (Argentina). We thank J. Sabio for linguistic revision of the manuscript.

\section{LITERATURE CITED}

1. Amiard, V., Demmig-Adams, B., Mueh, K. E., Turgeon, R., Combs, A. F., and Adams, W. W., III 2007. Role of light and jasmonic acid signaling in regulating foliar phloem cell wall ingrowth development. New Phytol. 173:722-731.

2. AOAC. 1999. Official Method 940.28. Fatty acids (free) in crude and refined oils. Vol. 2. Chapter 41:10 in: Official Methods of Analysis of AOAC International, 16th ed. P. Cunniff, ed. AOAC International, Gaithersburg, MD.

3. AOAC. 1999. Official Method 965.33. Peroxide value of oils and fats. Vol. 2. Chapter 41:9B in: Official Methods of Analysis of AOAC International, 16th ed. P. Cunniff, ed. AOAC International, Gaithersburg, MD.

4. Asis, R., Barrionuevo, D. L., Giorda, L. M., Nores, M. L., and Aldao, M. A. 2005. Aflatoxin Production in six peanut (Arachis hypogaea L.) genotypes infected with Aspergillus flavus and A. parasiticus, isolated from peanut production areas of Cordoba, Argentina. J. Agric. Food Chem. 53:9274-9280.

5. Bannenberg, G., Martinez, M., Hamberg, M., and Castresana, G. 2009. Diversity of the enzymatic activity in the lipoxygenase gene family of Arabidopsis thaliana. Lipids 44:85-95.

6. Bennett, J. W., and Klich, M. 2003. Mycotoxins. Clin. Microbiol. Rev.16:497-516.

7. Bradford, M. M. 1976. A rapid sensitive method for the quantification of microgram quantities of protein utilizing the principle of protein-dye binding. Anal. Biochem. 72:248-254.

8. Brash, A. R. 1999. Lipoxygenases: Occurrence, functions, catalysis, and acquisition of substrate. J. Biol. Chem. 274:23679-23682.

9. Brodhagen, M., Tsitsigiannis, D. I., Hornung, E., Goebel, C., Feussner, I., and Keller, N. P. 2008. Reciprocal oxylipin-mediated cross-talk in the Aspergillus-seed pathosystem. Mol. Microbiol. 67:378-391.

10. Burow, G. B., Gardner, H. W., and Keller, N. P. 2000. A peanut seed lipoxygenase responsive to Aspergillus colonization. Plant Mol. Biol. 42:689-701.

11. Burow, G. B., Nesbitt, T. C., Dunlap, J., and Keller. N. P. 1997. Seed lipoxygenase products modulate Aspergillus mycotoxin biosynthesis. Mol. Plant-Microbe Interact. 10:380-387.

12. Calvo, A. M., Hinze, L. L., Gardner, H. W., and Keller, N. P. 1999. Sporogenic effect of polyunsaturated fatty acids on development of Aspergillus spp. Appl. Environ. Microbiol. 65:3668-3673.

13. Christensen, S. A., and Kolomiets, M. V. 2011. The lipid language of plant-fungal interactions. Fungal Genet. Biol. 48:4-14.

14. Cleveland, T. E., Dowd, P. F., Desjardins, A. E., Bhatnagar, D., and Cotty, P. J. 2003. United States Department of Agriculture-Agricultural Research Service research on pre-harvest prevention of mycotoxins and mycotoxigenic fungi in U.S. crops. Pest Manage. Sci. 59:629-642.

15. Conover, W. J. 1999. Practical Nonparametric Statistics. John Wiley \& Sons, Inc., New York.

16. Dubbs, W. E., and Grimes, H. D. 2000. The mid-pericarp cell layer in soybean pod walls is a multicellular compartment enriched in specific lipoxygenase isoforms. Plant Physiol. 123:1281-1288.

17. Feussner, I., and Wasternack, C. 2002. The lipoxygenase pathway. Annu. Rev. Plant Physiol. Plant Mol. Biol. 53:275-297.

18. Fischer, A. M., Dubbs, W. E., Baker, R. A., Fuller, M. A., Stephenson, L. C., and Grimes, H. D. 1999. Protein dynamics, activity and cellular localization of soybean lipoxygenases indicate distinct functional roles for individual isoforms. Plant J. 19:543-554.

19. Fuller, M. A., Weichert, H., Fischer, A. M., Feussner, I., and Grimes, H. D. 2001. Activity of soybean lipoxygenase isoforms against esterified fatty acids indicates functional specificity. Arch. Biochem. Biophys. 388:146-154.

20. Gao, X., Brodhagen, M., Isakeit, T., Brown, S. H., Göbel, C., Betran, J., Feussner, I., Keller, N. P., and Kolomiets, M. V. 2009. Inactivation of the lipoxygenase ZmLOX3 increases susceptibility of maize to Aspergillus spp. Mol. Plant-Microbe Interact. 22:222-231.

21. Gardner, H. W. 1998. 9-Hydroxy-traumatin, a new metabolite of the lipoxygenase pathway. Lipids 33:745-749.

22. Grechkin, A. 1998. Recent developments in biochemistry of the plant lipoxygenase pathway. Prog. Lipid Res. 37:317-352.

23. Hayashi, S., Gresshoff, P. M., and Kinkema, M. 2008. Molecular analysis of lipoxygenases associated with nodule development in soybean. Mol. Plant-Microbe Interact. 21:843-853.

24. Howe, G. 2004. The role of hormones in defense against insects and 
disease. Pages 610-634 in: Plant Hormones Biosynthesis, Signal Transduction, Action! P. J. Davies, ed. Kluwer Academic Publishers, Dordrecht, The Netherlands.

25. Howe, G. A., and Schilmiller, A. L. 2002. Oxylipin metabolism in response to stress. Curr. Opin. Plant Biol. 5:230-236.

26. InfoStat. 2002. Grupo InfoStat Profesional. Facultad de Ciencias Agropecuarias, Universidad Nacional de Córdoba, Argentina.

27. Johnson, J. A., Blackburn, M. L., Bull, A. W., Welsch, C. W., and Watson, T. 1997. Separation and quantitation of linoleic acid oxidation products in mammary gland tissue from mice fed low and high fat diets. Lipids 32:369-375.

28. Kishimoto, K., Matsui, K., Ozawa, R., and Takabayashi, J. 2008. Direct fungicidal activities of C6-aldehydes are important constituents for defense responses in Arabidopsis against Botrytis cinerea. Phytochemistry 69:2127-2132.

29. Kisyombe, C. T., Beute, M. K., and Payne, G. A. 1985. Field evaluation of peanut genotypes for resistance to infection by Aspergillus parasiticus. Peanut Sci. 12:12-17.

30. Kombrink, E. 2012. Chemical and genetic exploration of jasmonate biosynthesis and signaling paths. Planta 236:1351-1366.

31. Maroufi, A., Bockstaele, E. V., and De Loose, M. 2010. Validation of reference genes for gene expression analysis in chicory (Cichorium intybus) using quantitative real-time PCR. BMC Mol. Biol. 11:15.

32. Mehan, V. K., McDonald, D., Nigam, S. N., and Lalitha, B. 1981. Groundnut cultivars with seed resistant to invasion by Aspergillus flavus. Oleagineux 30:501-507.

33. Mita, G., Gallo, A., Greco, V., Zasiura, C., Casey, R., Zacheo, G., and Santino, A. 2001. Molecular cloning and biochemical characterization of a lipoxygenase in almond (Prunus dulcis) seed. Eur. J. Biochem. 268:1500-1507.

34. Mixon, A. C., and Rogers, K. M. 1973. Peanut accessions resistant to seed infection by Aspergillus flavus. Agron. J. 65:560-562.
35. Porta, H., and Rocha-Sosa, M. 2002. Plant lipoxygenases. Physiological and molecular features. Plant Physiol. 130:15-21.

36. Prost, I., Dhondt, S., Rothe, G., Vicente, J., Rodriguez, M. J., Kift, N., and Fournier, J. 2005. Evaluation of the antimicrobial activities of plant oxylipins supports their involvement in defense against pathogens. Plant Physiol. 139:1902-1913.

37. Ridolfi, M., Terenziani, S., Patumi, M., and Fontanazza, G. 2002. Characterization of the lipoxygenases in some olive cultivars and determination of their role in volatile compounds formation. J. Agric. Food Chem. 50:835-839.

38. Tsitsigiannis, D. I., and Keller, N. P. 2006. Oxylipins act as determinants of natural product biosynthesis and seed colonization in Aspergillus nidulans. Mol. Microbiol. 59:882-892.

39. Tsitsigiannis, D. I., Kunze, S., Willis, D. K., Feussner, I., and Keller, N. P. 2005. Aspergillus infection inhibits the expression of peanut $13 \mathrm{~S}$ HPODE-forming seed lipoxygenases. Mol. Plant-Microbe Interact. 18: 1081-1089.

40. Upchurch, R. G. 2008. Fatty acid unsaturation, mobilization, and regulation in the response of plants to stress. Biotechnol. Lett. 30:967-977.

41. Vellosillo, T., Martínez, M., López, M. A., Vicente, J., Cascón, T., Dolan, L., Hamberg, M., and Castresana, C. 2007. Oxylipins produced by the 9-lipoxygenase pathway in Arabidopsis regulate lateral root development and defense responses through a specific signaling cascade. Plant Cell 19:831-846.

42. Veronico, P., Giannino, D., Melillo, M. T., Leone, A., Reyes, A., Kennedy, M. W., and Bleve-Zacheo, T. 2006. A novel lipoxygenase in pea roots. Its function in wounding and biotic stress. Plant Physiol. 141:10451055 .

43. Zeringue, H. J., Jr., Brown, R. L., Neucere, J. N., and Cleveland, T. E. 1996. Relationships between C6-C12 alkanal and alkenal volatile contents and resistance of maize genotypes to Aspergillus flavus and aflatoxin production. J. Agric. Food Chem. 44:403-407. 